INPLASY

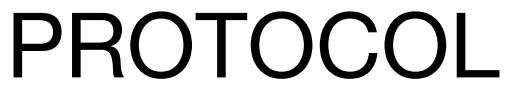

To cite: Gao et al. Prenatal phthalate exposure is associated with age-specific alterations in markers of adiposity in offspring: a systematic review. Inplasy protocol 202170090. doi: 10.37766/inplasy2021.7.0090

Received: 28 July 2021

Published: 28 July 2021

Corresponding author: Hui Gao

gh20190130@163.com

Author Affiliation:

The Affiliated Hospital of Anhui Medical University.

Support: 2020kj02, 2020xkj161 et al.

Review Stage at time of this submission: Formal screening of search results against eligibility criteria.

Conflicts of interest:

None declared.

\section{Prenatal phthalate exposure is associated with age-specific alterations in markers of adiposity in offspring: a systematic review}

Gao, $\mathrm{H}^{1}$; Wang, YF2; Wang, ZW3; Wang, Y4.

Review question / Objective: Population-Inclusion: Pregnant women (including occupational, high exposure from an environmental source). Exclusion: Non-pregnant women (including children, adolescent, elderly population, general population) and non-human mammalian animal species, etc. Exposure-Inclusion: Exposure to one or more of the phthalates or phthalate metabolites, as singular compounds or as mixtures, as determined by: 1) Measured concentration in contact medium (e.g., air, dust); 2) Biomarkers of exposure (e.g., urinary metabolite levels of phthalates); 3)Occupation involving exposure to phthalates (e.g., plastics manufacture); knowledge of specific contamination sites or accidental exposure. Exclusion: Not phthalate or its metabolite exposure. Comparison -Inclusion: A comparison population exposed to lower levels or no exposure/exposure below detection levels, or with different levels of target outcome group, or no target disease control group. Exclusion: other controls. OutcomeInclusion: Weight gain, body mass index, body fat and skin fold thickness measured at least at two time points from birth to later childhood. Exclusion: Other outcomes. Study designInclusion: observational study including cohort study and case-control study. Exclusion: a cross-sectional survey, review, systematic review, meta-analysis, comment, proceeding, etc.

INPLASY registration number: This protocol was registered with the International Platform of Registered Systematic Review and Meta-Analysis Protocols (INPLASY) on 28 July 2021 and was last updated on 28 July 2021 (registration number INPLASY202170090).

\section{INTRODUCTION}

Review question / Objective: PopulationInclusion: Pregnant women (including occupational, high exposure from an environmental source). Exclusion: Nonpregnant women (including children, adolescent, elderly population, general 
population) and non-human mammalian animal species, etc. Exposure-Inclusion: Exposure to one or more of the phthalates or phthalate metabolites, as singular compounds or as mixtures, as determined by: 1) Measured concentration in contact medium (e.g., air, dust); 2) Biomarkers of exposure (e.g., urinary metabolite levels of phthalates); 3)Occupation involving exposure to phthalates (e.g., plastics manufacture); knowledge of specific contamination sites or accidental exposure. Exclusion: Not phthalate or its metabolite exposure. Comparison -Inclusion: A comparison population exposed to lower levels or no exposure/ exposure below detection levels, or with different levels of target outcome group, or no target disease control group. Exclusion: other controls. Outcome-Inclusion: Weight gain, body mass index, body fat and skin fold thickness measured at least at two time points from birth to later childhood. Exclusion: Other outcomes. Study designInclusion: observational study including cohort study and case-control study. Exclusion: a cross-sectional survey, review, systematic review, meta-analysis, comment, proceeding, etc.

Condition being studied: The systematic literature search was performed in MEDLINE (accessed through PubMed), Web of Science, and CNKI (Chinese National Knowledge Infrastructure) until July, 2021. The inclusion and exclusion criteria following the PECOS (Population, Exposure, Comparison, Outcome, Study design) format were summarized. The data were extracted by two authors independently using a standardized spreadsheet. Two authors evaluated the risk of bias for adherence to the prespecified criteria. A conceptual framework was constructed to guide the organization and presentation of results based on the content analysis.

\section{METHODS}

Search strategy: MEDLINE: (phthalate OR phthalates OR PAEs OR PAE OR "phthalic acid ester" OR "phthalic diesters" OR "endocrine disruptor" OR "endocrine disrupting chemical") AND (growth OR "growth trajectories" OR "growth trajectory" OR "BMI trajectory" OR "body mass trajectory" OR "body mass trajectories" OR "trajectory of growth" OR anthropometric OR anthropometry OR obesity OR adiposity OR overweight OR "body mass" OR BMI OR "body size" OR "body fat" OR "body weight") AND (child OR children OR offspring OR childhood OR girl OR boy) AND humans[mh]. WEB OF SCIENCE: TS=((phthalate* OR "phthalic acid ester" OR "endocrine disruptor" OR "endocrine disrupting chemical") AND (growth OR "growth trajectories" OR "growth trajectory" OR "BMI trajectory" OR "body mass trajectory" OR "body mass trajectories" OR "trajectory of growth" OR anthropometric OR anthropometry OR obesity OR adiposity OR overweight OR "body mass" OR BMI OR "body size" OR "body fat" OR "body weight") AND (child OR children OR offspring OR childhood OR girl OR boy)) AND DT=Article. CNKI: $S U=$ ('邻苯二甲酸酯'+'环境内分泌干扰物') and $\mathrm{SU}=($ '生长'+'生长轨迹'+'BMI'+'体质指数'+'肥 胖'+'体重'+'体脂'+'体格') and SU=('儿童'+'子 代'+'男孩'+'女孩'+'男童'+'女童').

Participant or population: Inclusion: Pregnant women (including occupational, high exposure from an environmental source). Exclusion: Non-pregnant women (including children, adolescent, elderly population, general population) and nonhuman mammalian animal species, etc.

Intervention: Inclusion: Exposure to one or more of the phthalates or phthalate metabolites, as singular compounds or as mixtures, as determined by: 1) Measured concentration in contact medium (e.g., air, dust); 2) Biomarkers of exposure (e.g., urinary metabolite levels of phthalates); 3) Occupation involving exposure to phthalates (e.g., plastics manufacture); knowledge of specific contamination sites or accidental exposure. Exclusion: Not phthalate or its metabolite exposure.

Comparator: Inclusion: A comparison population exposed to lower levels or no exposure/exposure below detection levels, 
or with different levels of target outcome group, or no target disease control group. Exclusion: other controls.

Study designs to be included: Inclusion: observational study including cohort study and case-control study. Exclusion: a crosssectional survey, review, systematic review, meta-analysis, comment, proceeding, etc.

Eligibility criteria: (1)Population: Pregnant women (including occupational, high exposure from an environmental source); (2) Exposure: Exposure to one or more of the phthalates or phthalate metabolites, as singular compounds or as mixtures. (3) Comparison: A comparison population exposed to lower levels or no exposure/ exposure below detection levels, or with different levels of target outcome group, or no target disease control group. (4)Outcome: Weight gain, body mass index, body fat and skin fold thickness measured at least at two time points from birth to later childhood. (5) Study design: Observational study including cohort study and case-control study.

Information sources: The systematic literature search was performed in MEDLINE (accessed through PubMed), Web of Science, and CNKI (Chinese National Knowledge Infrastructure) until July, 2021.

Main outcome(s): Inclusion: Weight gain, body mass index, body fat and skin fold thickness measured at least at two time points from birth to later childhood. Exclusion: Other outcomes.

Quality assessment / Risk of bias analysis: Selection bias-Low: No evidence for selection bias. Authors found no difference in the background characteristics between the participants included and excluded from the analysis. Probably low: There is a little difference in characteristics between included and excluded participants. Probably high: Exclusions of high number of participants $(>30 \%)$ and no analysis to show this selection bias did not impact the results. High: Excluded samples have an impact on the results. Exposure assessment-Low: The exposures were assessed using validated methods, each analytical run included calibration standards, reagent blanks, and quality control samples. Probably low: The exposures were assessed using validated methods, but no information present on blank samples. Probably high: The methods of measuring exposure have not been elaborated. High: Non-standard methods does not been validated in other studies. Outcome assessment-Low: Body weight or fat or skin fold thickness was assessed using an international recognized instrument, assessments were carried out by at least one trained physician, authors reported the reliability and/or quality control process. Probably low: The authors used recognized tools to assess body weight or fat or skin fold thickness, testers were trained, but quality control checks were not described. Probably high: No information presented about the process of administering the assessment, the assessment instruments were common. High: Non-standard as sessment instruments does not appear validated in other studies, and no information presented about the process of administering the assessment. Confounding-Low: The studies controlled the necessary confounding factors: chid's gender, maternal pregnancy body mass index/overweight/obesity and gestational weight gain, breastfeeding, exercise, fat/ caloric intake. Probably low: The studies controlled four to six necessary confounders. Probably high: The numbers of necessary confounding factors controlled in the literature is less than four. High: The studies controlled none of confounding factors. Incomplete outcome data-Low: The outcome data was complete, missing values were imputed using appropriate methods. Or there was little missing data (30\%), and no argument is presented that would invalidate the possibility of selection bias. High: Only reporting on statistically significant findings, no information on the outcome data was reported. Selective outcome reporting-Low: All pre-specified outcomes 
were reported. Probably low: The authors only reported most of the outcomes, not all. Probably high: There are only statistical inferred results and no descriptive analysis results. High: The authors only reported statistically significant results. Other source of bias-Low: No other potential sources of bias identified. High: There were other bias.

Strategy of data synthesis: A conceptual framework was constructed to guide the organization and presentation of results based on the content analysis.

Subgroup analysis: None.

Sensitivity analysis: None.

Country(ies) involved: China (The Affiliated Hospital of Anhui Medical University).

Keywords: Phthalate, Prenatal exposure, Adiposity, Age-specific alteration.

Contributions of each author:

Author 1 - Hui Gao.

Email: gh20190130@163.com

Author 2 - Ya-fei Wang.

Email: 82716925@qq.com

Author 3 - Zi-wei Wang.

Email: 2937720843@qq.com

Author 4 - Yue Wang.

Email: 1059645873@qq.com 